\title{
The Experience of Robot-assisted Laparoscopic Transabdominal Preperitoneal Inguinal Hernia Repair Following Robot-assisted Laparoscopic Radical Prostatectomy: A Cohort Study
}

Naotake Funamizu ( $\square$ funamizujikei@yahoo.co.jp )

Ehime University

Sho Mineta

Ageo Central General Hospital

Takahiro Ozaki

Ageo Central General Hospital

Kohei Mishima

Ageo Central General Hospital

Kazuharu Igarashi

Ageo Central General Hospital

Kenji Omura

Ageo Central General Hospital

Yasutsugu Takada

Ehime University

Go Wakabayashi

Ageo Central General Hospital

\section{Research Article}

Keywords: inguinal hernia, laparoscopy, robotic surgical procedures, postprostatectomy inguinal hernia, robot-assisted laparoscopic transabdominal preperitoneal inguinal hernia repair (R-TAPP), robot-assisted laparoscopic radical prostatectomy (RALP)

Posted Date: January 24th, 2022

DOI: https://doi.org/10.21203/rs.3.rs-1274816/v1

License: (9) This work is licensed under a Creative Commons Attribution 4.0 International License.

Read Full License 


\section{Abstract}

\section{Background}

There are no reports of robot-assisted laparoscopic transabdominal preperitoneal inguinal hernia repair (R-TAPP) for the patients who underwent robot-assisted laparoscopic radical prostatectomy (RALP).

\section{Aim}

To evaluate the feasibility and safety of R-TAPP in patients who underwent RALP for postprostatectomy inguinal hernia $(\mathrm{PIH})$.

\section{Methods}

This is a prospective, single-center retrospective cohort study. R-TAPP was conducted in 74 consecutive patients from September 2016 to March 2020. Except for women and patients who underwent previous abdominal surgery, 70 patients were classified into two groups based on the absence or presence of PIH (PIH group or non-PIH group). The data were retrospectively compared to those who had not undergone RALP.

\section{Results}

Seventy patients were reviewed. Among them, 22.9\% had previously undergone RALP. We identified 22 lesions in 16 patients (unilateral in 10; bilateral in 6) in the PIH group, and 67 lesions in 54 patients (unilateral in 41; bilateral in 13) in the non-PIH group. The PIH group showed a longer median operation time compared to the non-PIH group. However, postoperative complications-including seroma formation, hematoma, and surgical site infections-were not significantly different between the groups. There were no recorded vascular, bladder, or spermatic cord injuries in either group. There was no conversion to laparoscopic or open surgery in either group. The estimated blood loss was small, and hospitalization duration was one day in all cases. Moreover, there were no hernia recurrences within the 90-day follow-up period in either group.

\section{Conclusion}

R-TAPP seem to be a feasible and safe approach for inguinal hernia repair, even in patients who underwent RALP for prostate cancer.

\section{Introduction}

Robot-assisted surgery using the da Vinci system is widely accepted in several organ diseases compared to laparoscopic surgery [1-3]. In particular, the robot system has been proactively introduced in urological diseases [4-6]. Robot-assisted laparoscopic radical prostatectomy (RALP) is a prevailing surgical technology for the treatment of prostate cancer. However, radical prostatectomy (RP) can result 
in the development of postprostatectomy inguinal hernias $(\mathrm{PIH})$, the estimated incidence rates of which were reported as $4.2 \%-15.9 \%$ following surgery $[7,8]$. A recent report showed that TAPP for PIH is potentially reliable without prolonging the duration of operation [9]. However, the appropriate approach and repair method of the surgical procedure for $\mathrm{PIH}$ remains controversial because of surgical difficulty arising from preperitoneal adhesion. The appropriate approach for PIH is still unknown. This study therefore evaluated the feasibility and safety of robot-assisted laparoscopic transabdominal preperitoneal inguinal hernia repair (R-TAPP) in patients who had PIH following RALP compared to those who did not undergo RALP.

\section{Materials And Methods}

\section{Study design}

The study protocol was reviewed and approved by the Ethics Committee of Ageo Central General Hospital (approval number: AMG935, 2021), and the study was conducted in accordance with the Helsinki Declaration as revised in 2013. The inclusion criteria for this study were R-TAPP cases for inguinal hernia in men without previous abdominal surgery excluding RALP and who had PIH following RALP. The exclusion criteria were as follows: (1) women, (2) patients who previously underwent abdominal surgery and (3) patients who had recurrent inguinal hernias (Fig 1). The primary endpoint of the present study was to evaluate the safety of R-TAPP for PIH after RALP compared to R-TAPP for inguinal hernia. Seventy-four patients (male: female $=71: 3$ ) who underwent R-TAPP were registered at Ageo Central General Hospital between September 2016 and October 2020. Of these, four patients were women and/or underwent previous abdominal surgery or had recurrent hernias and were excluded from this study. The patients were divided into two groups based on the presence of PIH following RALP. Thus, final analysis was performed on 16 with PIH (PIH group) and 54 without PIH patients (non-PIH group). The two groups were retrospectively reviewed and compared. The analyzed perioperative outcomes included operation time $(\mathrm{min})$, estimated blood loss $(\mathrm{mL})$, duration of postoperative hospital stay (day), conversion to open surgery, intra- or postoperative complications such as surgical site infections and intestinal injury-based on the Clavien-Dindo classification [10] (CD), and recurrence. TAPP or R-TAPP are the standard treatment for the inguinal hernia in our hospital. R-TAPP procedure was recommended for all patients with inguinal hernia at the first visit. However, R-TAPP was performed only for those who had accepted personal expense, because the Japanese universal health insurance system still has not approved robot-assisted surgery for inguinal hernia at present in Japan. We obtained written informed consent for medical treatment due to R-TAPP from all patients. But the patients provided no written informed consent for the use of their data in clinical research, because this study is retrospective study. Thus, the patient and their data were not anonymized before analysis. The follow-up period was a minimum of 10-postoperative months in both groups (Figure 1).

\section{Operative technique for $R$-TAPP}


The patient was placed in the Trendelenburg position under general anesthesia. The da Vinci® X or Xi surgical system (Intuitive Surgical Inc.) was used and docked with the patient from the left side. Three robotic arms were used including one for the camera and two for robotic instruments. The camera trocar was placed at the umbilical area with a 30-degree camera. Two other robotic trocars were localized $9 \mathrm{~cm}$ apart from the umbilicus (each site) at the level of the umbilicus (Figure 2). Surgical devices included Cadiere forceps in the left hand and Maryland bipolar forceps in the right hand. Initially, a $15 \mathrm{~cm}$ transverse peritoneal incision was made $4-5 \mathrm{~cm}$ above the internal inguinal ring (Figure 3A). The hernia sac was completely dissected from the spermatic cord and reversed into the abdominal cavity. Cooper's ligament was dissected and identified carefully after reaching the psoas muscle and pubic symphysis (Figure 3B). The spermatic cord and vas deferens were carefully confirmed and preserved. A lateral dissection of the preperitoneal space was then required to insert a mesh. In all cases, a selffixating mesh (Parietex ProGrip ${ }^{\circledR}$, Medtronic) was used. This mesh comprises of anatomical monofilament polyester (Figure 3C). The fixation of the mesh was shortened to avoid postoperative neural pain. In case of bilateral inguinal hernia repair, the peritoneal incision was extended on the opposite side. Finally, the peritoneum was closed with continuous sutures using monofilament thread (30 Prolene ${ }^{\circledR}$, Ethicon) (Figure 3D).

\section{Statistical analysis}

All statistical analyses were performed using GraphPad Prism v5.0 (GraphPad Software Inc., La Jolla, CA, USA) and SPSS version 24 (SPSS Inc., Chicago, IL, USA). Continuous data were expressed as median range. Categorical data were expressed as number and percentages. The statistical analyses were determined using Student's t-test, $\chi^{2}$ test, or Fisher's exact probability test, as appropriate. P-value of $<0.05$ was considered to indicate a statistically significant difference. A multivariate analysis, based on the logistic regression model, was used to identify whether PIH was a risk factor for postoperative complications following R-TAPP. P-value of $<0.05$ was considered statistically significant.

\section{Results}

Seventy registered patients were reviewed. According to the Japan Hernia Society Classification [11], our cohort included type I hernias in 69 patients, type II in 13 patients, and type IV in 7 patients. Also, there were 51 patients with unilateral hernias and 19 patients with bilateral hernias. There were 16 patients with PIH (PIH group) and 54 patients without PIH (non-PIH group). There were no differences in variables such as age, American Society of Anesthesiologists (ASA) classification, and body mass index (BMI) (Table 1). Twenty-nine patients underwent R-TAPP on the right, 22 on the left, and 19 bilaterally (Fig 4). Table 2 shows perioperative outcomes following R-TAPP. The median operation time was 180.4 and $123.4 \mathrm{~min}$ in the $\mathrm{PIH}$ and non-PIH group, respectively $(\mathrm{P}=0.001)$. Additionally, for the unilateral inguinal hernias, the median operation time was $167.2 \mathrm{~min}$ (range: $123-247 \mathrm{~min}$ ) in the $\mathrm{PIH}$ group and $114.9 \mathrm{~min}$ (range: $51-204 \mathrm{~min})$ in the non-PIH group $(P=0.001)$. For the bilateral inguinal hernias, the median operation time was $202.5 \mathrm{~min}$ (range: 152-306 min) and $149.2 \mathrm{~min}$ (range: 103-251 min) in the PIH and non-PIH groups, respectively $(P=0.018)(F i g)$. All R-TAPP procedures were completed without 
conversion to open or laparoscopic approach in both groups. Additionally, all patients had tackless mesh fixation to avoid the risk of postoperative pain. Peritoneal closure was accomplished with 4-0 Polydioxanone (PDS) monofilament suture (Ethicon Inc.) with sequential running sutures using one or two threads. With the exception of one patient with a hematoma (CD classification-I) in the non-PIH group, no patient experienced postoperative complications or chronic pain according to the CD classification. Moreover, no recurrence was seen in either group within at least three months.

\section{Discussion}

RALP has become a commonplace surgical approach for local prostate cancer over the last decade [12]. Recent evidence demonstrated that RALP was associated with better intraoperative outcomes compared to laparoscopic radical prostatectomy (LRP) [13], although high volume center became a requirement for better outcomes [14]. However, RP including RALP sometimes leads to PIH, which is painful and affects the patient's quality of life. The reported incidence rate of PIH was $3.4 \%-7.3 \%$ after RALP and $8.4 \%$ after open RP $[15,16]$. PIH occurs within the first three years after RP [17]. A large nationwide population-based study also demonstrated that no significant difference was found regarding the risk of PIH between RP and RALP in 11,212 patients [18]. Generally, patients who previously underwent robot-assisted surgery tend to desire robot-assisted surgery for another surgery. The present study therefore aimed to evaluate whether R-TAPP was available for PIH.

Well-known risk factors for developing PIH include older age, previous inguinal hernia repair, and low BMI $[19,20]$. Moreover, Otaki et al. showed that psoas muscle volume $(P M V)<350 \mathrm{~cm}^{3}$ was an independent risk factor for PIH [21]. Additionally, Iwamoto et al. showed that dilatation of the internal inguinal ring represents an important risk factor for PIH [22]. The precise mechanism of developing PIH is still unknown, but it is definitive that a surgical procedure is a crucial factor in the development of PIH. A presently accepted theory is that a potentially existing vaginal process of the peritoneum becomes clinically evident because of peritoneal dissection around the internal inguinal ring [23]. Therefore, indirect inguinal hernia is the most frequent hernia type of $\mathrm{PIH}[24,25]$. There is present data to support the relevance of this hypothesis.

Regarding prophylactic methods, Kadono et al. reported that dissection of the peritoneum from the internal inguinal ring, and separating the spermatic cord and vessels from the peritoneum, could reduce the incidence of PIH following RALP; however, they did not acquire statistical significance [26]. In contrast, Iwamoto et al. described creating an incisional line of peritoneum as an important prophylactic method. They proposed that incising the peritoneum sufficiently close to the internal inguinal ring could prevent PIH [22].

Regarding treatment in patients with histories of pelvic or abdominal surgeries including RP inguinal hernia repair, such as transabdominal preperitoneal (TAPP) and totally extra peritoneal (TEP) repair, may be difficult because of the need to dissect peritoneal adhesions, unlike in patients without surgical histories. Izadipani et al. showed that L-TAPP for PIH has good results and is effective [27]. In addition, 
Dulucq et al. reported that L-TEPP for PIH can be performed efficiently and safely in patients after RP by skilled and experienced laparoscopic surgeons [28]. Moreover, Sakon et al. showed that L-TAPP inguinal hernia repair after RALP was comparably safer and more effective than open surgery. They concluded that L-TAPP repair may be a valuable alternative to open hernioplasty [29]. In contrast, Angus et al. showed that R-TAPP might be an alternative to open repair in patients with a history of prior urologic pelvic operation [30]. Additionally, Bitnner and Leblanc et al. reported that operation times were longer for robotic hernia repair patients than laparoscopic patients, but there was no difference in the safety $[31,32]$. Under such conditions, the safety of R-TAPP for PIH has not been established. To our knowledge, there is no evidence that R-TAPP is a useful and safe procedure for PIH. Thus, in this study, we reviewed 16 cases of R-TAPP for PIH and evaluated their surgical outcomes in comparison with 54 cases of RTAPP for non-PIH. R-TAPP was first introduced in our department in September 2016 under the mentorship of professor Kudsi [33]. Thus, we perform R-TAPP according to his instructions. The point to ponder is that R-TAPP is still not covered by Japanese health insurance. Therefore, surgical cost will become an obstacle to patients with inguinal hernias and thereby make patient recruitment for R-TAPP difficult in Japan. However, several institutions have gradually shown evidence of spread of R-TAPP in Japan [34].

The fact that R-TAPP is a more suitable surgical technique for PIH may lead to its increased demand and popularity. On the other hand, Prabhu et al. exhibited no clinical benefit to the robotic approach for straightforward inguinal hernia repair compared with the laparoscopic approach. They concluded that the robotic approach incurred higher costs and longer operation time compared with the laparoscopic approach [35]. Additionally, Kohraki et al. revealed that the outcomes of L-TAPP were significantly superior to the R-TAPP and at lower hospital costs [36]. In contrast, Forrester et al. reported that short-term quality of life after R-TEPP was improved compared to open and laparoscopic repairs. Thus, although the relative merit by approach for inguinal hernia repair is still controversial, we believe that the clinical benefit of robotic surgery is the multiarticular function of ease of performing peritoneal suturing compared to L-TAPP. However, our study has several noteworthy limitations. First, the relatively small number of PIH cases may cause a lack of statistical power. Next, our data were from a retrospective cohort study and collected at a single hospital. Thereafter, a more comprehensive prospective study should be conducted to confirm our findings in the near future.

\section{Conclusion}

In conclusion, RALP for prostate cancer is gradually becoming a prevalent procedure. When PIH occurs after RALP, patients are more likely to hope for a similar procedure using a robotic system. We believe that R-TAPP might be a feasible and safe approach, even in cases of PIH.

\section{Declarations}

\section{Acknowledgements}


None

\section{Funding}

We have no funding support.

\section{Author contributions}

Naotake Funamizu designed this study. Sho Mineta, Takahiro Ozaki, Kohei Mishima, and Kazuharu Igarashi collected data. Kenji Omura and Yasutsugu Takada contributed to analyze the data. Go Wakabayashi contributed to its critical revision. All the authors read and approved the final manuscript.

\section{Availability of data and materials}

The data is available by contacting the authors.

\section{Ethics approval and consent to participate}

Institutional review board statement: This study was reviewed and approved by the Ethics Committee of Ageo Central General Hospital (approval number: AMG935, 2021).

\section{Consent for publication}

Patients were not required to give informed consent to the study because the analysis used anonymous clinical data that were obtained after each patient agreed to treatment by written consent. For full disclosure, the details of the study are published on the home page of Ageo Central General Hospital.

\section{Competing interests}

We have no competing interests to disclose.

Data sharing statement: No additional data are available.

\section{References}

1. Zhang L, Yuan Q, Xu Y, Wang W. Comparative clinical outcomes of robot-assisted liver resection versus laparoscopic liver resection: A meta-analysis. PLOS ONE 2020; 15: e0240593 [doi: 10.1371/journal.pone.0240593, PMID 33048989]

2. Wang Y, Liu Y, Han G, Yi B, Zhu S. The severity of postoperative complications after robotic versus laparoscopic surgery for rectal cancer: A systematic review, meta-analysis and meta-regression. PLOS ONE 2020; 15: e0239909 [doi: 10.1371/journal.pone.0239909, PMID 33002066]

3. Di Martino M, Caruso R, D'Ovidio A, et al. Robotic versus laparoscopic distal pancreatectomies: A systematic review and meta-analysis on costs and perioperative outcome. Int J Robot 2021; 3 : e2295. 
4. Li H, Liu C, Zhang H, Xu W, Liu J, Chen Y, Li T, Li B, Wu Z, Xia T. The Use of Unidirectional Barbed Suture for urethrovesical anastomosis during Robot-Assisted radical Prostatectomy: A Systematic Review and Meta-Analysis of Efficacy and Safety. PLOS ONE 2015; 10:e0131167 [doi: 10.1371/journal.pone.0131167, PMID 26135310]

5. Marzorati C, Monzani D, Mazzocco K, Pavan F, Cozzi G, De Cobelli O, Monturano M, Pravettoni G. Predicting trajectories of recovery in prostate cancer patients undergone Robot-Assisted Radical Prostatectomy (RARP). PLOS ONE 2019; 14: e0214682 [doi: 10.1371/journal.pone.0214682, PMID 30946773]

6. Kim DK, Kim LH, Raheem AA, Shin TY, Alabdulaali I, Yoon YE, Han WK, Rha KH. Comparison of Trifecta and Pentafecta Outcomes between T1a and T1b Renal Masses following Robot-Assisted Partial Nephrectomy (RAPN) with Minimum one year follow up: can RAPN for T1b Renal Masses Be Feasible? PLOS ONE 2016; 11: e0151738 [doi: 10.1371/journal.pone.0151738, PMID 26987069]

7. Zhu S, Zhang H, Xie L, Chen J, Niu Y. Risk factors and prevention of inguinal hernia after radical prostatectomy: A systematic review and meta-analysis. J Urol 2013; 189: 884-890 [doi: 10.1016/j.juro.2012.08.241, PMID 23009871]

8. Ku JY, Lee CH, Park WY, Lee NK, Baek SH, Ha HK. The cumulative incidence and risk factors of postoperative inguinal hernia in patients undergoing radical prostatectomy. Int J Clin Oncol 2018; 23: 742-748 [doi: 10.1007/s10147-018-1244-0, PMID 29340839]

9. Ohuchi M, Fukunaga M, Nagakari K, Azuma D, Kohama S, Nomoto J, Sakamoto K. Surgical technique and outcomes of transabdominal preperitoneal inguinal hernia repair after radical prostatectomy: dissection between the transversalis fascia and superficial layers of preperitoneal fascia. Hernia 2019; 23:167-174 [doi: 10.1007/s10029-018-1800-6, PMID 30047042]

10. Dindo D, Demartines N, Clavien PA. Classification of surgical complications: A new proposal with evaluation in a cohort of 6336 patients and results of a survey. Ann Surg 2004; 240: 205-213 [doi: 10.1097/01.sla.0000133083.54934.ae, PMID 15273542]

11. Japanese Hernia Society. Japanese Hernia Society classification of inguinal hernia; 2009. Available from: http://www.med.teikyo-u.ac.jp/ surgery2/hernia (updated Apr 2009], [accessed Jan 24 2020]).

12. Saika T, Miura N, Fukumoto T, Yanagihara Y, Miyauchi Y, Kikugawa T. Role of robot-assisted radical prostatectomy in locally advanced prostate cancer. Int J Urol 2018; 25: 30-35 [doi: 10.1111/iju.13441, PMID 28901630]

13. Huang $X$, Wang $L$, Zheng $X$, Wang $X$. Comparison of perioperative, functional, and oncologic outcomes between standard laparoscopic and robotic-assisted radical prostatectomy: A systemic review and meta-analysis. Surg Endosc 2017; 31: 1045-1060 [doi: 10.1007/s00464-016-5125-1, PMID 27444830]

14. Godtman RA, Persson E, Cazzaniga W, Sandin F, Carlsson S, Ahlgren G, Johansson E, Robinsson D, Hugosson J, Stattin P. Association of surgeon and hospital volume with short-term outcomes after robot-assisted radical prostatectomy: nationwide, population-based study. PLOS ONE 2021; 16: e0253081 [doi: 10.1371/journal.pone.0253081, PMID 34138904] 
15. Lee KS, Koo KC, Chung BH. Long-term results of the plugging method with regard to the prevention of a postoperative inguinal hernia after robot-assisted laparoscopic prostatectomy: A retrospective study. J Endouro/ 2017; 31: 1183-1188 [doi: 10.1089/end.2017.0340, PMID 28931316]

16. Nilsson H, Stranne J, Hugosson J, Wessman C, Steineck G, Bjartell A, Carlsson S, Thorsteinsdottir T, Tyritzis SI, Lantz A, Wiklund P, Haglind E. Risk of hernia formation after radical prostatectomy: a comparison between open and robot-assisted laparoscopic radical prostatectomy within the prospectively controlled LAPPRO trial. Hernia 2020:1-8 [doi: 10.1007/s10029-020-02178-7, PMID 32279170]

17. Abe T, Shinohara N, Harabayashi T, Sazawa A, Suzuki S, Kawarada Y, Nonomura K. Postoperative inguinal hernia after radical prostatectomy for prostate cancer. Urology 2007; 69: 326-329 [doi: 10.1016/j.urology.2006.09.043, PMID 17275071]

18. Fridriksson JÖ, Folkvaljon Y, Lundström KJ, Robinson D, Carlsson S, Stattin P. Long-term adverse effects after retropubic and robot-assisted radical prostatectomy. Nationwide, population-based study. J Surg Oncol 2017; 116: 500-506 [doi: 10.1002/jso.24687, PMID 28591934]

19. Liu L, Xu H, Qi F, Wang S, Shadhu K, Ramlagun-Mungur D, Wang S. Incidence and risk factors of inguinal hernia occurred after radical prostatectomy-comparisons of different approaches. $B M C$ Surg 2020; 20: 218 [doi: 10.1186/s12893-020-00883-9, PMID 33008371]

20. Rabbani F, Yunis LH, Touijer K, Brady MS. Predictors of inguinal hernia after radical prostatectomy. Urology 2011; 77: 391-395 [doi: 10.1016/j.urology.2010.04.019, PMID 20646748]

21. Otaki T, Hasegawa M, Yuzuriha S, Hanada I, Nagao K, Umemoto T, Shimizu Y, Kawakami M, Nakajima N, Kim H, Nitta M, Hanai K, Kawamura Y, Shoji S, Miyajima A. Clinical impact of psoas muscle volume on the development of inguinal hernia after robot-assisted radical prostatectomy. Surg Endosc 2021; 35: 3320-3328 [doi: 10.1007/s00464-020-07770-7, PMID: 32720178]

22. Iwamoto H, Morizane S, Hikita K, Honda M, Takenaka A. Postoperative inguinal hernia after roboticassisted radical prostatectomy for prostate cancer: evaluation of risk factors and recommendation of a convenient prophylactic procedure. Cent Eur J Uro/ 2019; 72: 418-424 [doi: 10.5173/ceju.2019.0002, PMID 32015914]

23. Fukuta F, Hisasue S, Yanase M, Kobayashi K, Miyamoto S, Kato S, Shima M, Tsukamoto T, Takatsuka K. Preoperative computed tomography finding predicts for postoperative inguinal hernia: new perspective for radical prostatectomy-related inguinal hernia. Urology 2006; 68: 267-271 [doi: 10.1016/j.urology.2006.02.023, PMID 16904432]

24. Regan TC, Mordkin RM, Constantinople NL, Spence IJ, Dejter SW. Incidence of inguinal hernias following radical retropubic prostatectomy. Urology 1996; 47: 536-537 [doi: 10.1016/S00904295(99)80491-9, PMID 8638364]

25. Sakai Y, Okuno T, Kijima T, Iwai A, Matsuoka Y, Kawakami S, Kihara K. Simple prophylactic procedure of inguinal hernia after radical retropubic prostatectomy: isolation of the spermatic cord. Int $J$ Urol 2009; 16: 848-851 [doi: 10.1111/j.1442-2042.2009.02370.x, PMID 19811549] 
26. Kadono Y, Nohara T, Kawaguchi S, Sakamoto J, Iwamoto H, Yaegashi H, Nakashima K, lijima M, Shigehara K, Izumi K, Mizokami A. Novel prevention procedure for inguinal hernia after robotassisted radical prostatectomy: results from a prospective randomized trial. J Endourol 2019; 33: 302-308 [doi: 10.1089/end.2018.0898, PMID 30767563]

27. Izadpanahi MH, Milasi R, Izadpanahi MH. Evaluation of the results and complications of transabdominal preperitoneal laparoscopic inguinal hernia repair in patients with a history of radical prostatectomy. Urol J 2020;17: 24-29 [doi: 10.22037/uj.v0i0.4751, PMID 30345490]

28. Dulucq JL, Wintringer $P$, Mahajna A. Totally extraperitoneal (TEP) hernia repair after radical prostatectomy or previous lower abdominal surgery: is it safe? A prospective study. Surg Endosc 2006; 20: 473-476 [doi: 10.1007/s00464-006-3027-3, PMID 16463074]

29. Sakon M, Sekino Y, Okada M, Seki H, Munakata Y. Laparoscopic inguinal hernioplasty after robotassisted laparoscopic radical prostatectomy. Hernia 2017; 21: 745-748 [doi: 10.1007/s10029-0171639-2, PMID 28799065]

30. Angus A, DeMare A, lacco A. Evaluating outcomes for robotic-assisted inguinal hernia repair in males with prior urologic surgery: A propensity-matched analysis from a national database. Surg Endosc 2020: 35; 5310-5314 [doi: 10.1007/s00464-020-08020-6, PMID 33006032]

31. Bittner IV JG, Cesnik LW, Kirwan T, Wolf L, Guo D. Patient perceptions of acute pain and activity disruption following inguinal hernia repair: A propensity-matched comparison of robotic-assisted, laparoscopic, and open approaches. J Robot Surg 2018; 12: 625-632 [doi: 10.1007/s11701-0180790-9, PMID: 29453731]

32. LeBlanc K, Dickens E, Gonzalez A, Gamagami R, Pierce R, Balentine C, Voeller G, Prospective Hernia Study Group. Prospective, multicenter, pairwise analysis of robotic-assisted inguinal hernia repair with open and laparoscopic inguinal hernia repair: early results from the Prospective Hernia Study. Hernia 2020; 24: 1069-1081 [doi: 10.1007/s10029-020-02224-4, PMID 32495043]

33. Kudsi OY, McCarty JC, Paluvoi N, Mabardy AS. Transition from laparoscopic totally extraperitoneal inguinal hernia repair to robotic transabdominal preperitoneal inguinal hernia repair: A retrospective review of a single surgeon's experience. World J Surg 2017; 41: 2251-2257 [doi: 10.1007/s00268-0173998-3, PMID 28337532]

34. Saito T, Fukami Y, Uchino T, Kurahashi S, Matsumura T, Osawa T, Arikawa T, Komatsu S, Kaneko K, Sano T. Preliminary results of robotic inguinal hernia repair following its introduction in a singlecenter trial. Ann Gastroenterol Surg 2020; 4: 441-447 [doi: 10.1002/ags3.12341, PMID 32724888]

35. Prabhu AS, Carbonell A, Hope W, Warren J, Higgins R, Jacob B, Blatnik J, Haskins I, Alkhatib H, Tastaldi L, Fafaj A, Tu C, Rosen MJ. Robotic inguinal vs transabdominal laparoscopic inguinal hernia repair: the rival randomized clinical trial. JAMA Surg 2020; 155: 380-387 [doi: 10.1001/jamasurg.2020.0034, PMID 32186683]

36. Khoraki J, Gomez PP, Mazzini GS, Pessoa BM, Browning MG, Aquilina GR, Salluzzo JL, Wolfe LG, Campos GM. Perioperative outcomes and cost of robot-assisted versus 
laparoscopic inguinal hernia repair. Surg Endosc 2020; 34: 3496-3507 [doi: 10.1007/s00464-01907128-8, PMID 31571036]

\section{Tables}

Due to technical limitations, table 1 and 2 is only available as a download in the Supplemental Files section.

Figures

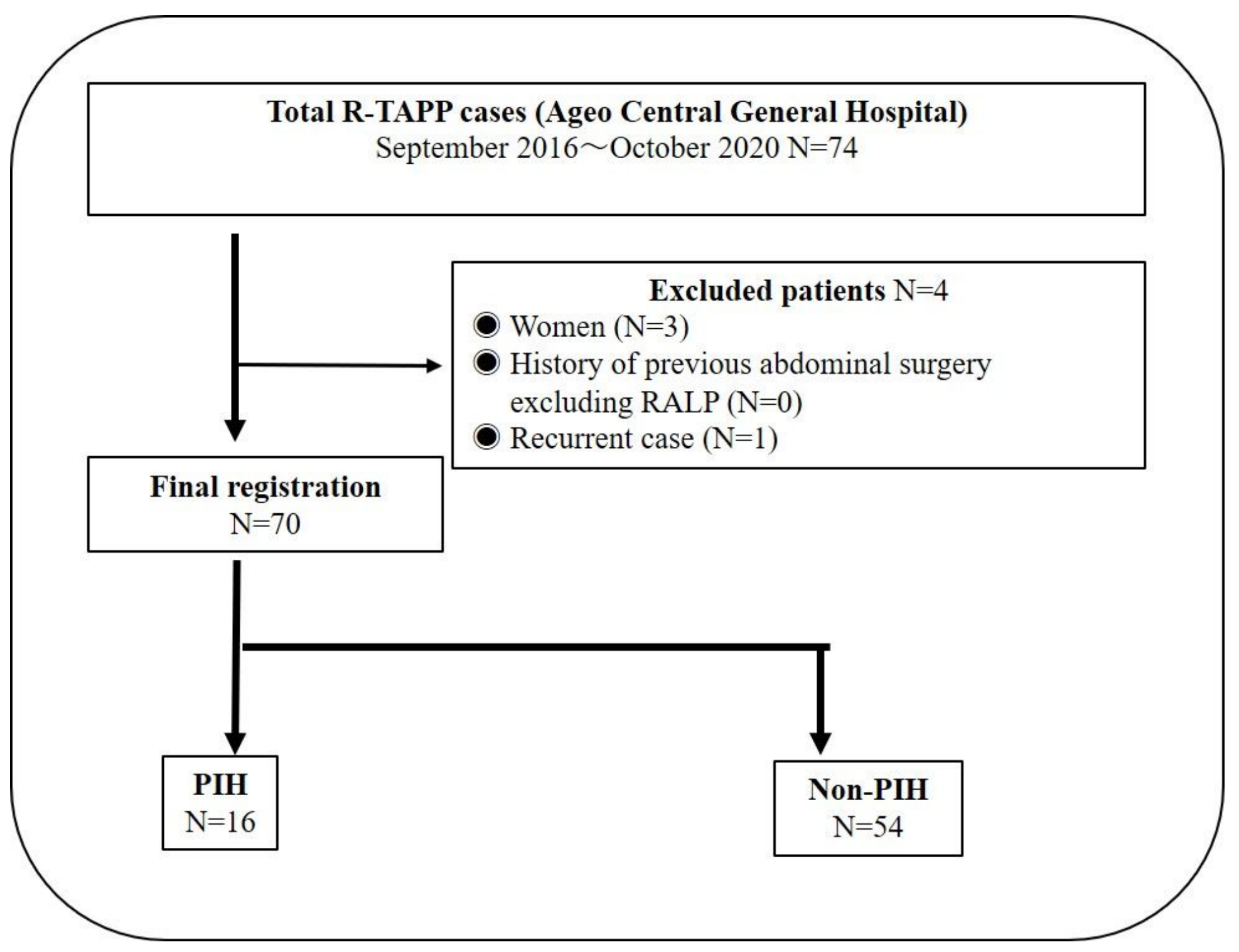

Figure 1

Flowchart of study population 


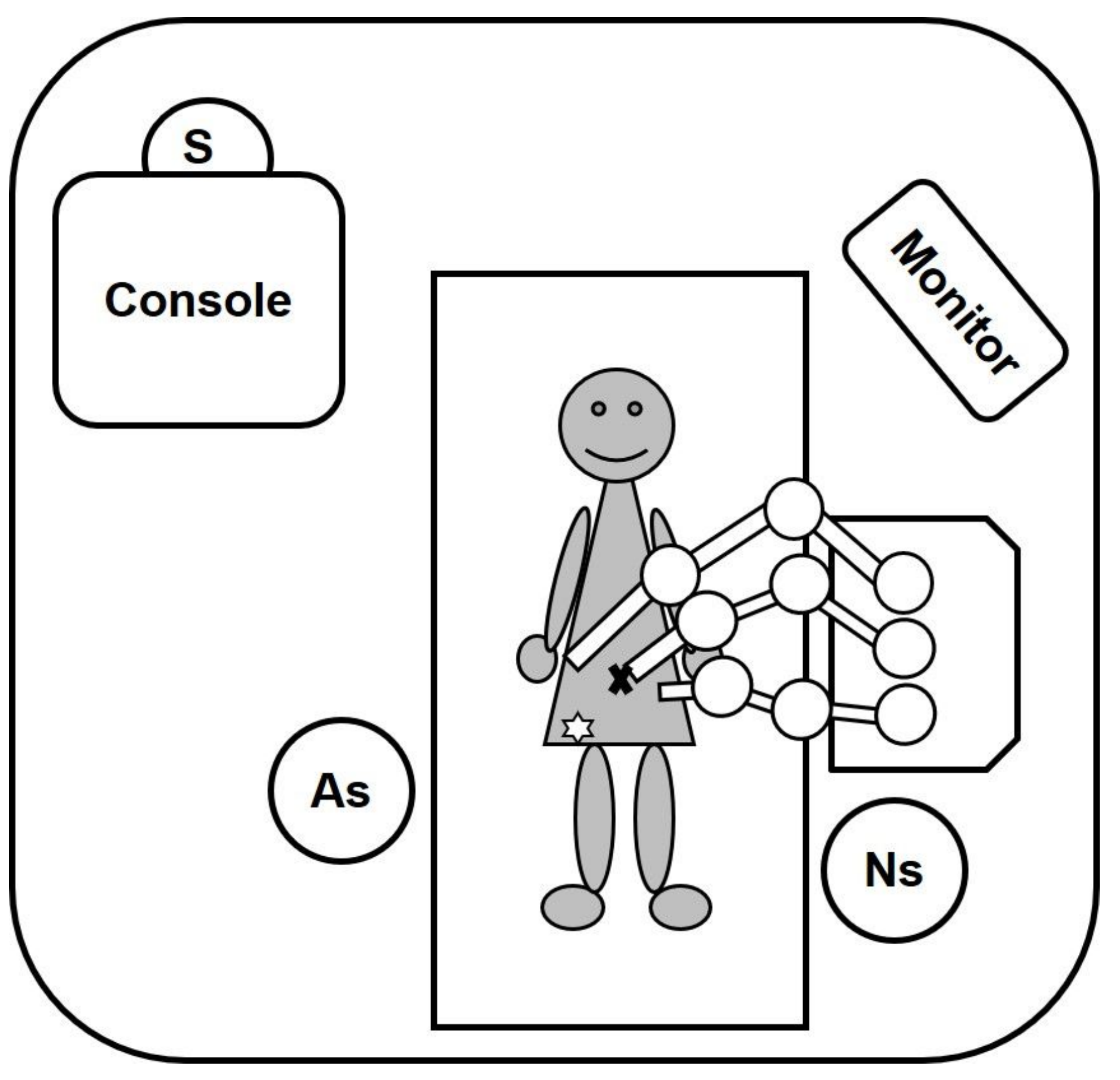

Figure 2

Layout diagram 


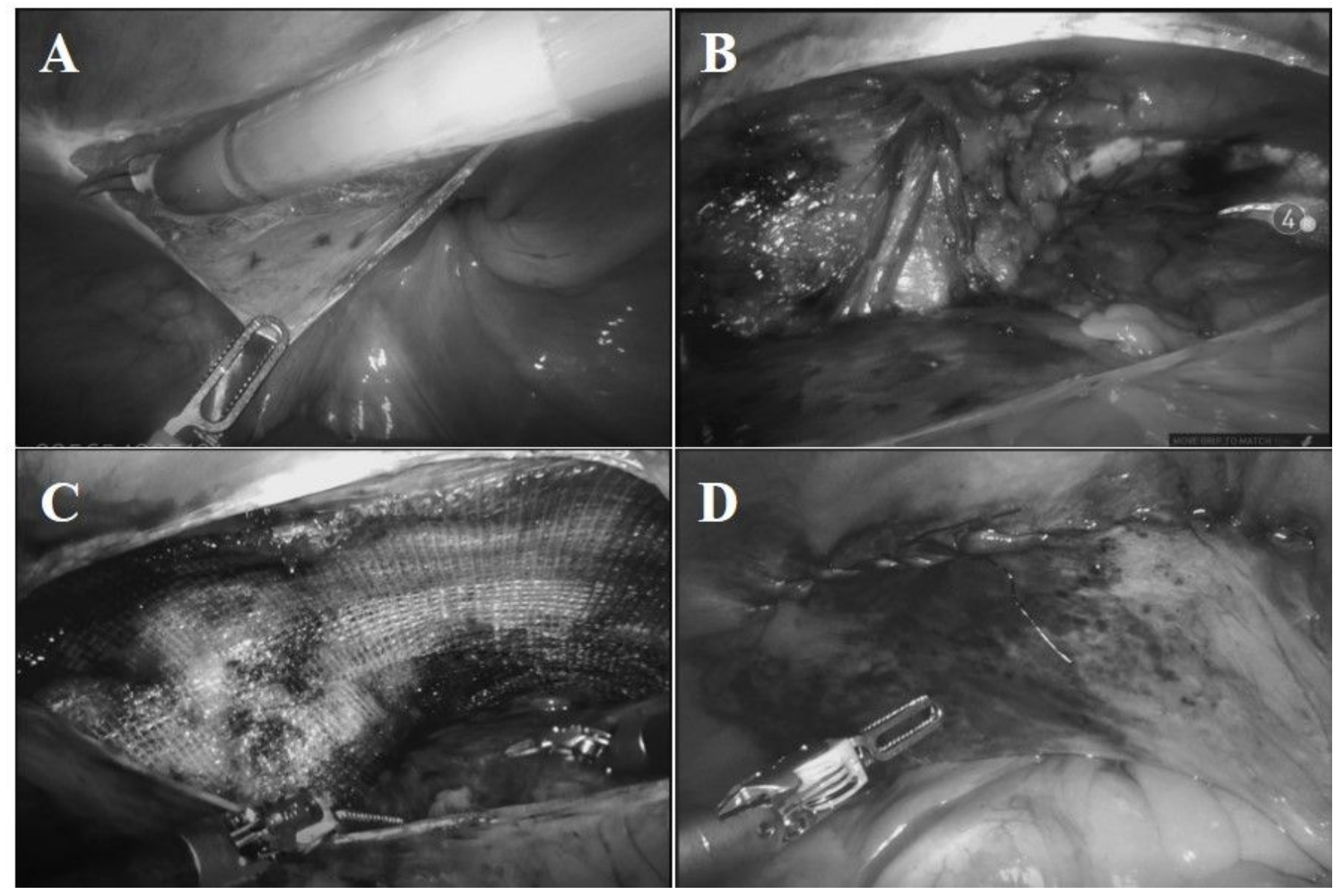

Figure 3

\section{Surgical procedure}

(A) The peritoneum is laid open $4-5 \mathrm{~cm}$ above the internal inguinal ring. (B) The hernia sac is dissected without injury of the spermatic cord and testicular vessels. (C) After securing the peritoneal flap, the Parietex ProGrip ${ }^{\mathrm{TM}}$ is placed. (D) Open peritoneum is closed by suturing using monofilament thread. 


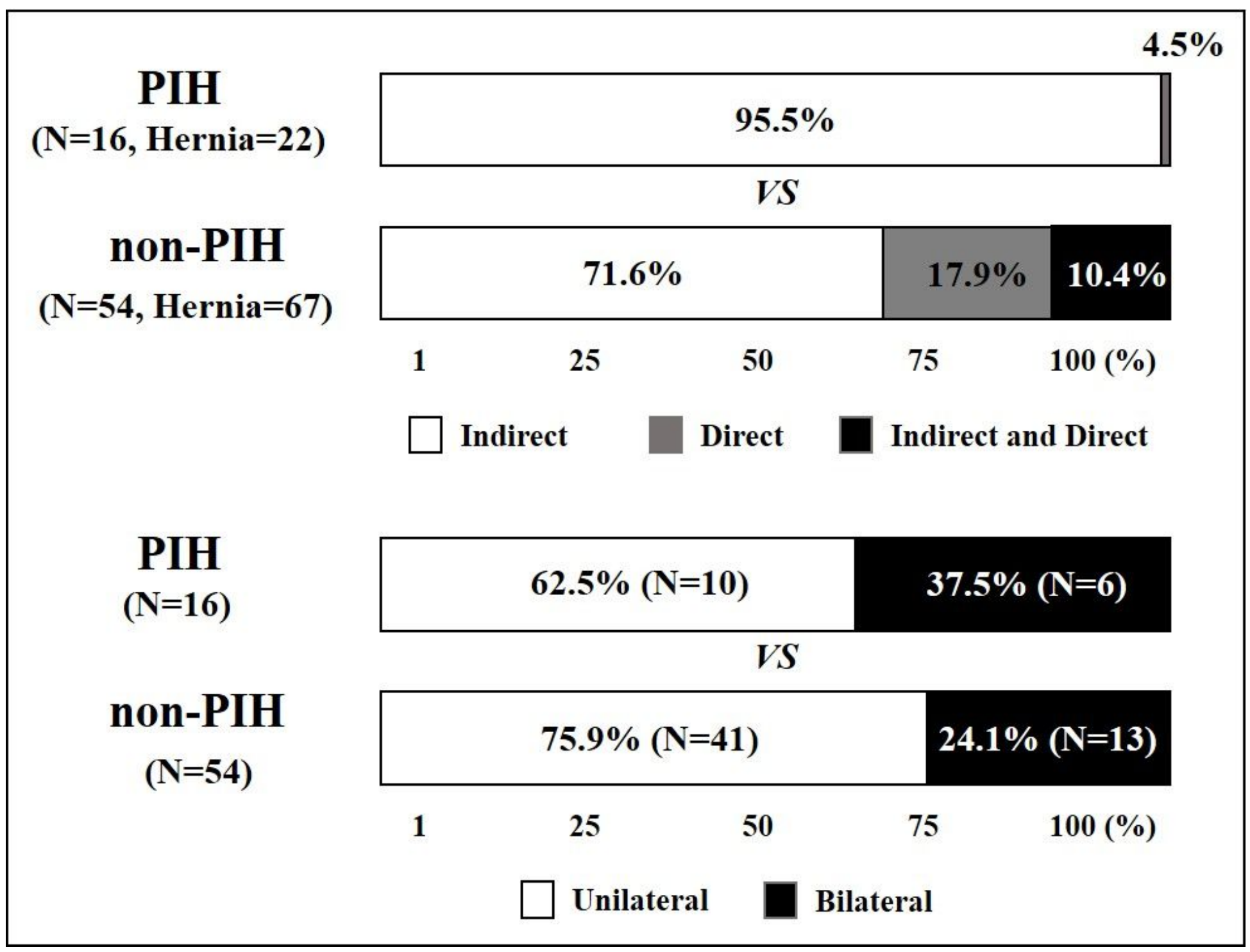

Figure 4

Rate of hernia type and side 


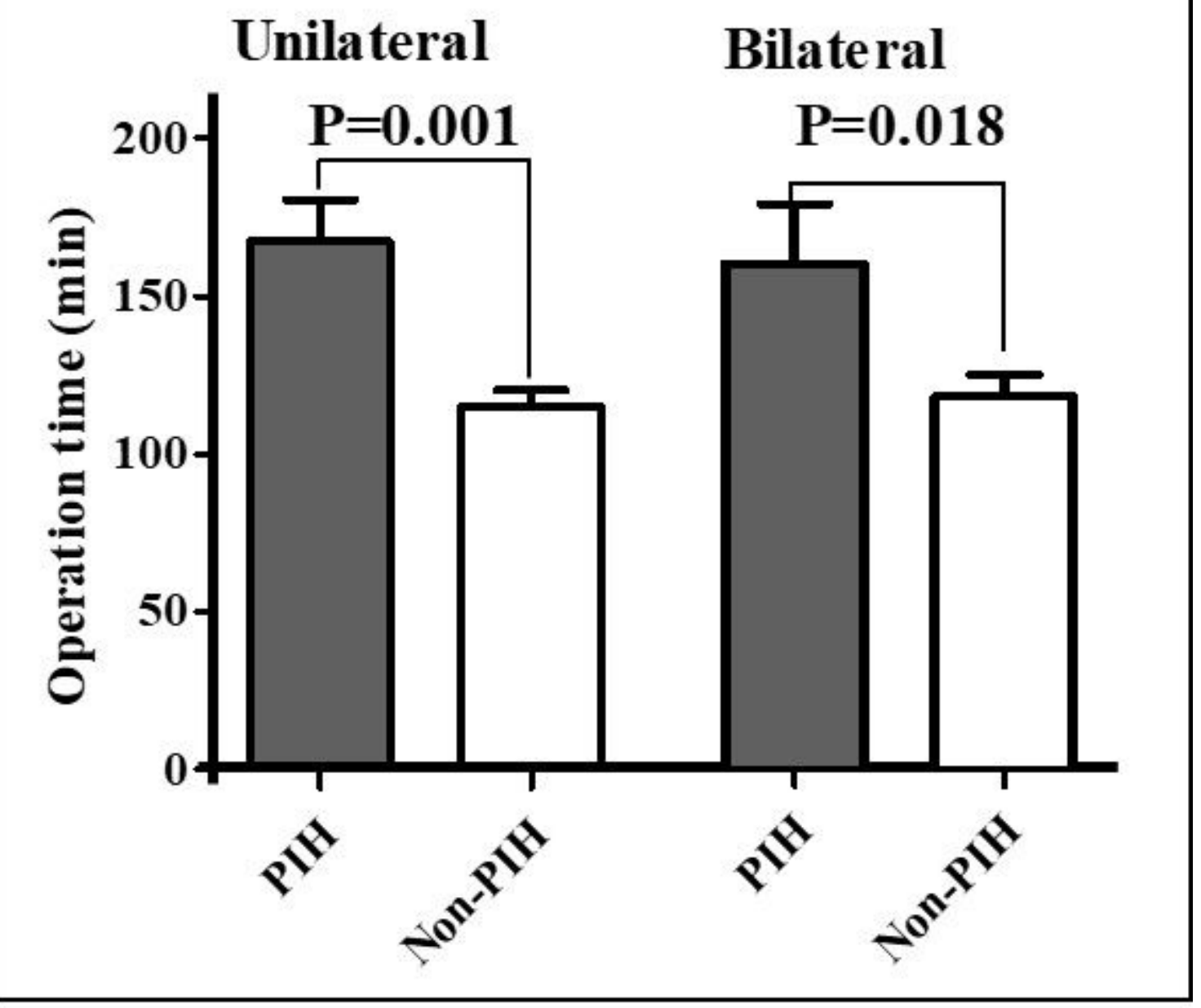

Figure 5

Comparison of operation type according to hernia number

\section{Supplementary Files}

This is a list of supplementary files associated with this preprint. Click to download.

- FinalFileTable1.xlsx

- FinalFileTable2.xlsx 